\title{
A Flexible Simple Thermostat for Small Objects and the Range of 100 to $400 \mathrm{~K}$
}

\author{
H. R. BILGe ANM M-A. Nicolet \\ California Institute of Technology, Pasadena, California 91109 \\ (Received 8 September 1969; and in final form, 12 November 1969)
}

\begin{abstract}
A flexible, inexpensive thermostat for the temperature range 100 to $400 \mathrm{~K}$ is described. Liquid nitrogen is the coolant and a gas serves as transfer medium. The temperature can be set to better than $1 \mathrm{~K}$ and is held there within $\frac{1}{2} \mathrm{~K}$ by an electronic control system. The relatively small working volume of the order $10 \mathrm{~cm}^{3}$ allows quick changes of temperature, a desirable feature in typical semiconductor experiments.
\end{abstract}

A NUMBER of thermostats have been described in the recent literature. Some of them confine the temperature fluctuations within the test chamber to less than $10^{-2}$ or $10^{-3} \mathrm{~K}$ by sophisticated mechanical designs or advanced electronic control systems, or both. ${ }^{1-6}$ Others are designed to meet specific purposes ${ }^{7-11}$ covering temperatures ranging from $4.2 \mathrm{~K}$ to high values. Those of Refs. 10 and 11 operate with a streaming gas as temperature bath. Compared to the more conventional methods based on static thermal equilibria, this principle offers the advantages of speed in response, high flexibility in layout, and intimate thermal contact with the object under investigation independently of its mechanical support. These advantages are significant particularly for small objects with internal heat dissipation, such as are encountered frequently when studying electrical phenomena in solids.

The thermostat described in this paper combines the features of the streaming gas as temperature bath with those of an electronic control of moderate sophistication. The result is a general purpose inexpensive system. To permit its construction by simple means, vacuum isolation is avoided and only commonly available materials are used. The system meets the following specifications: (i) a temp-

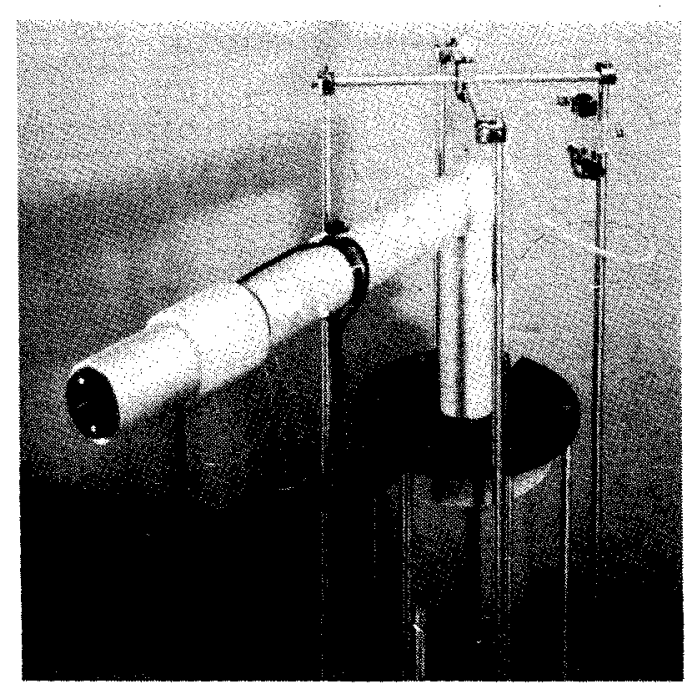

Fic. 1. General view of the thermostat (without the electronic control system). The working volume is located at the left, with front cover removed, to reveal the opening of the chamber. perature range extending approximately from 100 to $400 \mathrm{~K}$, (ii) a stability and reproducibility of about $1 \mathrm{~K}$, (iii) a modest active volume of the order of $10 \mathrm{~cm}^{3}$, and (iv) a simple and flexible design which can easily be adapted to varying experimental conditions such as access by light, short electrical connections for fast electronic pulses, magnetic fields, etc. We feel that these conditions are frequently encountered in other laboratories and thus report here on the main features of our thermostat. In contrast to Refs. 10 and 11, a suitable electronic regulator is also described. This control system adjusts the temperature to any preselected value within a few minutes and also insures the long term stability.

A complete description of the whole system, with drawings, diagrams, parts list, and suppliers, is made available elsewhere (see final paragraph).

\section{DESCRIPTION OF THE SYSTEM}

Figure 1 shows a picture of the thermostat. A 4 liter Dewar with wide neck contains liquid $\mathrm{N}_{2}$. Purified air or nitrogen gas from an external supply flows through spirals of copper tubing immersed in the liquid. The cold gas is transferred to the thermostat chamber through a brass tube which is thermally insulated against the room by three layers of $1.27 \mathrm{~cm}$ thick polyethylene foam and one outer layer of $6.4 \mathrm{~mm}$ thick sheet of the same material. This transfer tube is rigidly attached to a phenolic plate. The plate itself is clamped onto the top of the Dewar and serves as a tight lid to it. A hole in the lid serves both as a vent for the evaporating nitrogen and for refilling liquid $\mathrm{N}_{2}$ during long runs. In Fig. 1 the transfer tube has the shape of an elbow so as to reach between the poles of a magnet or over the top of a bench. The whole unit can be raised or lowered with a little jack and moved on four cartwheels (below bottom of the figure, not visible).

The thermostat chamber, housing the heater and the active volume, is attached to the end of the transfer tube with a phenolic flange. Polyethylene also surrounds this joint (located under the thickened segment of polyethylene in Fig. 1) and the chamber assembly (open-ended head section of polyethylene in Fig. 1). With this layout, different head assemblies are easily interchanged to suit different needs. The object under study is attached to a front cover of 
phenolic which seals off the chamber with an O-ring. The front cover is thermally insulated with polyethylene as well. (This cover is removed in Fig. 1 to show the chamber opening.) A sensing thermocouple in good thermal contact with the object is also inserted into the chamber through the cover, as well as other leads and feedthroughs necessary in the experiment. The sensing thermostat controls an electronic regulator which provides electrical power to the heater. For fast response to changes in the due temperature, the wall chambers are made of thin brass tubing and the heater consists of printed circuit type meanders of a thin metal strip on a flexible heat resistant substrate about $2.5 \mathrm{~cm}$ wide and $11.4 \mathrm{~cm}$ long. This substrate is wound in a spiral at the inner end of the chamber. The cold gas is forced to circulate within the spiral before entering the chamber proper. The electrical connections to the heater and a supplementary thermocouple are visible in Fig. 1 too.

The gas leaves the chamber through holes located towards the front in the thin brass wall. (The holes are too small to be seen in the photograph.) The gas then flows back along the outer side of the wall before escaping to the outside through the tube pointing downwards in Fig. 1. This reverse-flow arrangement improves the uniformity of the temperature within the chamber and cuts down the requirements on gas consumption. A straight pattern of flow can also be adopted, at some loss of performance. This eliminates the double wall of the chamber and simplifies the mechanical design still further. For operation solely above room temperatures the Dewar is left empty.

The electronic control system contains a commercial component oven for the reference junction of the thermocouple. A temperature compensated Zener diode provides a standard from which a voltage is derived whose value equals that expected from the thermocouple at the thermostat temperature desired. This voltage is subtracted from the actual emf of the thermocouple, the difference is amplified up to about $10^{6}$ times and fed back into the heater. For convenience, the potentiometer selecting the voltage is referenced of a virtual electrical $0^{\circ} \mathrm{C}$ point and is equipped with a digital dial calibrated in millivolts. Standard thermocouple tables can then be used directly to set the temperature.

\section{DESCRIPTION OF PERFORMANCE}

The highest temperature accessible is given by the softening point of the polyethylene foam (about $90^{\circ} \mathrm{C}$ ). Higher temperatures can be reached easily by inserting a layer of more heat resistant insulator between the walls of the thermostat chamber and the foam. The lowest temperature is determined by the acceptable consumption of cooling gas. The cooling capacity of the gas decreases to zero at $77 \mathrm{~K}$ and the operation at temperatures of $100 \mathrm{~K}$ and below becomes increasingly wasteful. In that range, a cold finger arrangement is superior to convective cooling with gas.

Short time fluctuations (minutes and below) measured on a piece of brass weighing a few grams are typically confined to an interval of $\frac{1}{2} \mathrm{~K}$ over the whole temperature range of the system. Long term fluctuations (hours and days) lie within the same limits. The setting of the temperature has an absolute error of approximately $1 \mathrm{~K}$. In operation, the thermostat consumes typically 1 liter $/ \mathrm{h}$ of liquid nitrogen, 600 to 700 liters/h of (nitrogen) gas, and a maximum of $70 \mathrm{~W}$ at full heater power. It takes about 5 min for the new steady state to be reached when the temperature is increased by $100 \mathrm{~K}$, and about twice as long for a downward step of the same magnitude.

A complete description of the thermostat, with drawings, diagrams, parts lists, and suppliers, will be available upon request from the NASA Scientific and Technical Information Facility, P.O. Box 33, College Park, Maryland 20740. We thank Dr. J. Hopkins, NASA Electronics Research Center, who encouraged us in pursuing this work. The financial support under NGR 05-002-100 of the NASA Electronics Research Center, Cambridge, Massachusetts, is also very gratefully acknowledged.

* On leave from Oklahoma State University, Stillwater, Okla. 74074 .

${ }_{1}^{1}$ A. van Itterbeek et al., Cryogenics 4, 8 (1964).

${ }^{2}$ N. I. Davidenko and I. G. Fakidov, Instrum. Exp. Tech. 5, 1239

(1967).

${ }^{3} \mathrm{~J}$. Bigeleisen et al., Rev. Sci. Instrum. 39, 353 (1968).

${ }^{4} \mathrm{~S}$. Arajs and J. W. Conroy, Japan. J. Appl. Phys. 8, 614 (1969).

${ }^{5} \mathrm{~J}$. Hennephof $e t$ al., J. Sci. Instrum. 2, 96 (1969)

${ }^{6}$ C. P. Pickup and W. R. G. Kemp, Cryogenics 9, 90 (1969).

${ }^{7}$ D. McSweeney et al., Rev. Sci. Instrum. 36, 1324 (1965).

${ }^{8}$ B. Sharon and D. Treves, Rev. Sci. Instrum. 37, 1252 (1966).

${ }^{9}$ C. R. Duda, Rev. Sci. Instrum. 39, 1484 (1968).

${ }^{10}$ H. K. Kessler, Rev. Sci. Instrum. 37, 517 (1966).

W. P. Knox and J. D. Cuthbert, Rev. Sci. Instrum. 39, 1181 (1968). 